\title{
Perfil clínico-epidemiológico de pacientes con endocarditis infecciosa, período 2003-2010 en el hospital de Temuco, Chile
}

\author{
BENJAMÍN STOCKINS ${ }^{1}$, VICTOR NEIRA ${ }^{1}$, ALEJANDRO PAREDES $^{1}$, \\ CARLOS CASTILLO ${ }^{2}$, ANDRÉS TRONCOSO $^{\mathrm{a}}$
}

${ }^{1}$ Universidad de La

Frontera - Hospital Dr Hernán Henríquez Aravena Temuco, Chile

${ }^{2}$ Hospital de Los Angeles

anterno de Medicina, Universidad de La Frontera.

Recibido el 27 de diciembre de 2011, aceptado el 15 de junio de 2012

Correspondencia a:

Dr. Benjamín Stockins Departamento. de

Medicina Interna

Facultad de Medicina, Universidad de La Frontera Manuel Montt 112, Temuco.

\section{Profile of patients with infective endocarditis admitted to a Chilean regional hospital}

Background: Mortality due to infective endocarditis (IE) in Chile is close to 30\%. Aim: To report the experience with patients admitted with the diagnosis of IE in a regional tertiary hospital. Material and Methods: Retrospective study of 107 patients aged $50 \pm 16$ years (75\% males) discharged with a definitive diagnosis of IE according to modified DUKE criteria, between years 2003 and 2010. Demographic variables, severity scores, clinical characteristics, bacteriology and hospital evolution were recorded. Results: Fifty nine percent of patients had concomitant cardiovascular problems. APACHE II and Sequential Organ Failure Assessment (SOFA) scores on admission were $8.4 \pm 4.7$ and $2.7 \pm 2.8$ respectively. Native valves were affected in $91 \%$ of cases (aortic and mitral valves in $62 \%$ and $50 \%$ of cases respectively). Prosthetic valves were affected in $9.3 \%$ of cases. Rheumatic heart disease was the predominant primary lesion in $10 \%$ of patients. Antibiotics were used in $45.1 \%$ before blood cultures were performed. In 68\% of patients blood cultures were positive. S. viridans (30.8\%), S.aureus (18.6\%) and coagulase negative Streptocicci (5.6\%) were the identified microorganisms. Intensive care unit admission was required in $48 \%$ of patients. Renal, heart and neurological deterioration was observed in 53, 34 and $14 \%$ of patients, respectively. Twenty percent of patients developed systemic embolism and $37 \%$ required heart surgery. Mean hospital stay was $28.3 \pm 19.1$ days and $27 \%$ of patients died. Conclusions: In this series of patients, IE has a high mortality. Most patients studied were admitted in bad conditions.

(Rev Med Chile 2012; 140: 1304-1311).

Key words: Echocardiography; Endocarditis, bacterial; Hospital mortality; Prognosis.
L a endocarditis infecciosa (EI) es una afección grave, que afecta al endocardio, especialmente a nivel valvular y que requiere de sospecha diagnóstica y tratamiento precoz por su alta morbi-mortalidad ${ }^{1}$. Tiene variadas formas de presentación y evolución, caracterizadas por un compromiso multiorgánico, dado por la agresividad del agente infeccioso causante ${ }^{2}$.

Característicamente existe formación de vegetaciones en la superficie de las válvulas cardíacas y más infrecuentemente en las cuerdas tendíneas, pared de cavidades y defectos interventriculares.

Los agentes más frecuentes son el estreptococo viridans y el estafilococo ${ }^{3}$, pero en forma progresiva se describen nuevos gérmenes. El origen del foco infeccioso o una maniobra causal no se identifican en la mayor parte de los pacientes ${ }^{4}$.

La incidencia en la población general permanece entre 1,7 a 6,2 por 100.000 habitantes/año en Europa y Estados Unidos de Norteamérica ${ }^{5}$. 
Estudios chilenos muestran una incidencia de 2-3 casos por 100.000 habitantes/ año, siendo una afección prevalente, con elevada morbilidad y una mortalidad que asciende a $25 \%$ en las series extranjeras y a 29\% en Chile ${ }^{6}$.

La terapia antibiótica tiene un rol crucial en el control y la eliminación de los gérmenes del organismo y del aparato cardiovascular. A pesar de esto, se requiere frecuentemente de la cirugía tanto para corregir la hemodinamia consecuente al daño valvular, como para erradicar el foco infeccioso ${ }^{7}$. Su desenlace sin tratamiento antibiótico es fatal en prácticamente todos los casos.

El presente trabajo presenta la realidad y el estado actual de esta patología en un centro terciario de referencia a nivel regional.

\section{Material y Método}

Revisamos el banco de datos del Centro Cardiovascular del Hospital de Temuco en el período 2003-2010. Se identificaron 107 pacientes (100\%) egresados con diagnóstico de EI según criterios de Duke modificados definitivos ${ }^{2,8}$.

La Tabla 1 muestra las variables medidas que comprenden datos demográficos, patología de base, capacidad funcional, severidad del cuadro al ingreso, medida por puntaje APACHE II (Acute Physiology And Chronic Health Evaluation) y SOFA (Sepsis-related Organ Failure Assessment). Asimismo, el compromiso valvular, uso de antibióticos, necesidad de cirugía, válvula comprometida, manejo intensivo, falla sistémica, presencia de embolías, resultado de cultivos, días de hospitalización y condición al egreso.

Los pacientes fueron tratados en salas de Medicina Interna y Unidades de Paciente Crítico y los esquemas de tratamiento antibiótico fueron los recomendados por normas nacionales e internacionales ${ }^{3}$.

Se consideró como origen reumático el tener el antecedente de la enfermedad o la presencia de un componente de estenosis en la válvula mitral.

Los datos fueron registrados en una planilla Epi Info ${ }^{\circledR}$ versión 3.3.2. El análisis estadístico fue hecho en Stata ${ }^{\circledR} 9.0$.
Tabla 1. Variables estudiadas

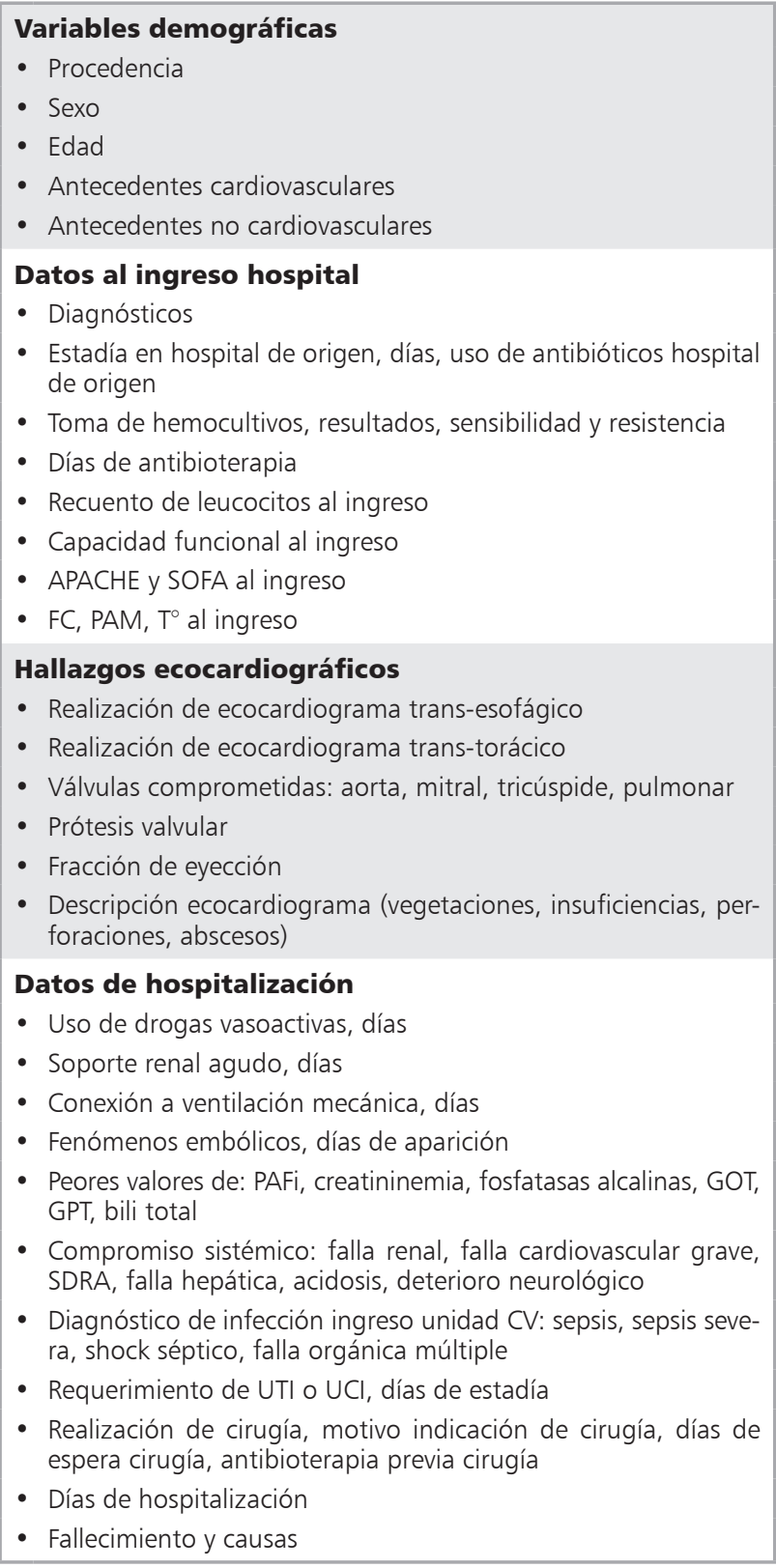

\section{Resultados}

\section{Características de la población}

Durante el período 2003-2010, 107 pacientes cumplieron con los criterios de Duke definitivos de EI. Sus características se presentan en la Tabla 2. El 66,2\% de los pacientes se presentó con fiebre asociada a un soplo 
cardíaco. En más del 90\% de los pacientes la endocarditis se asentó sobre una válvula nativa. En el resto fue en una válvula protésica y en un caso, en un cortocircuito cardíaco. No hubo compromiso del endocardio parietal y en un caso la etiología fue relacionada a drogadicción endovenosa. El 42,6\% de los pacientes presentaba al ingreso insuficiencia cardíaca en CF III o IV.

El 28,9\% de los pacientes procedía de hospitales periféricos, con una estadía media de 8 días en dichos centros. El 45,1\% usó antibióticos previo a la toma de hemocultivos.

Ningún paciente tuvo el antecedente de una maniobra susceptible de provocar bacteremia, sin embargo en 11 pacientes se diagnosticaron focos infecciosos concomitantes al ingreso: Seis casos con neumonia, una sacroileítis, un absceso cutáneo, un absceso dental, y dos infecciones del tracto urinario.

\section{Compromiso valvular y microbiología}

La Figural muestra la distribución según compromiso cardiaco, predominando las válvulas aórtica y mitral. En 23 pacientes hubo compromiso de más de una válvula $(21,4 \%)$. Las infecciones de válvulas protésicas fueron tardías en la mayoría de los casos (más de un año tras la cirugía).

El paciente con antecedentes de drogadicción tuvo compromiso de la válvula pulmonar.

En 68,3\% de los pacientes se aisló germen, predominando el S. viridans y S. aureus (Tabla 3 ). Hubo un caso de Kingella Kingae y uno de Actinomyces.

\section{Ecocardiografía}

Todos los pacientes fueron sometidos a ecocardiografía transtorácica y sus hallazgos fueron:

- Vegetaciones valvulares: 74,7\%.
Tabla 2. Descripción de la población en estudio

\begin{tabular}{|lc|}
\hline Variable & \\
\hline Sexo masculino & $74,7 \%$ \\
\hline Edad (años) & $49,9 \pm 16,4$ \\
\hline Procedencia urbana & $73,8 \%$ \\
\hline Comorbilidades & \\
- HTA & $41,1 \%$ \\
- DM tipo 2 & $16,8 \%$ \\
- Enfermedad reumática inactiva & $10,2 \%$ \\
- IRC en hemodiálisis & $3,7 \%$ \\
\hline ICC CF II-III ingreso & $74,5 \%$ \\
Compromiso válvula nativa & $90,6 \%$ \\
\hline
\end{tabular}

HTA = Hipertensión arterial. DM = Diabetes mellitus. IRC = Insuficiencia renal crónica. ICC = Insuficiencia cardíaca congestiva.

Tabla 3. Microorganismos aislados

\begin{tabular}{|lc|}
\hline Microorganismos aislados & Frecuencia (\%) \\
\hline Estreptoco viridans & $30,8 \%$ \\
\hline Estafilococo aureus & $18,6 \%$ \\
\hline Otros & $13,3 \%$ \\
\hline Estafilococo coagulasa (-) & $5,6 \%$ \\
\hline Hemocultivos negativos & $31,7 \%$ \\
\hline
\end{tabular}

- Ruptura valvular: 9,3\%.

- Abscesos: 6,5\%.

- Perforaciones valvulares: $4,6 \%$.

- Leak en válvulas protésicas: $3,7 \%$.

Se realizó ecocardiografía transesofágica en el 38,9\% de los casos. En la mayor parte de ellos fue por no tener un diagnóstico concluyente con

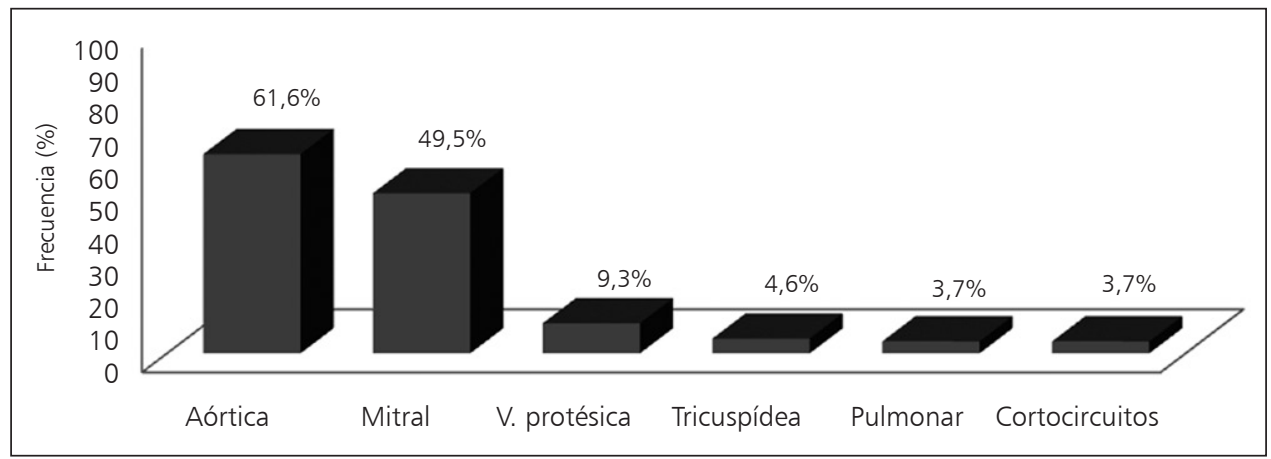

Figura 1. Compromiso cardíaCO 
la ecocardiografía transtorácica, existir un ecocardiograma de superficie negativo, para excluir complicaciones y para completar el estudio. El ecocadiograma transesofágico aportó $18,7 \%$ de vegetaciones así como 4,7\% de abscesos no diagnosticados con el eco transtoráxico.

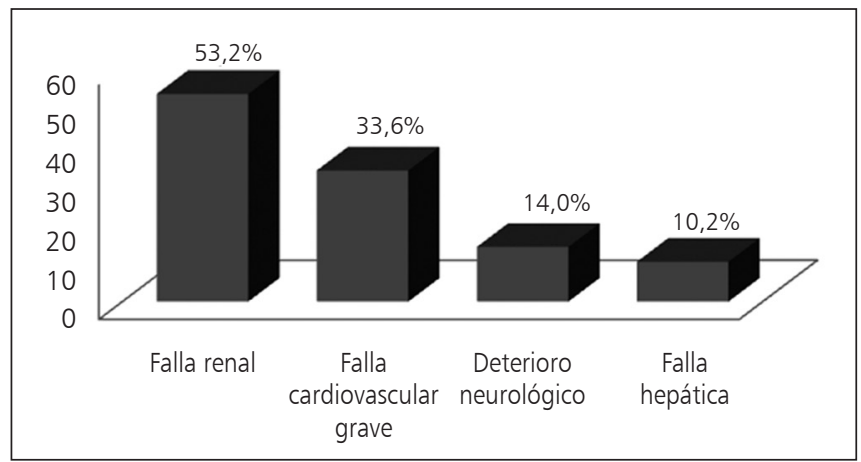

Figura 2. Compromiso sistémico.

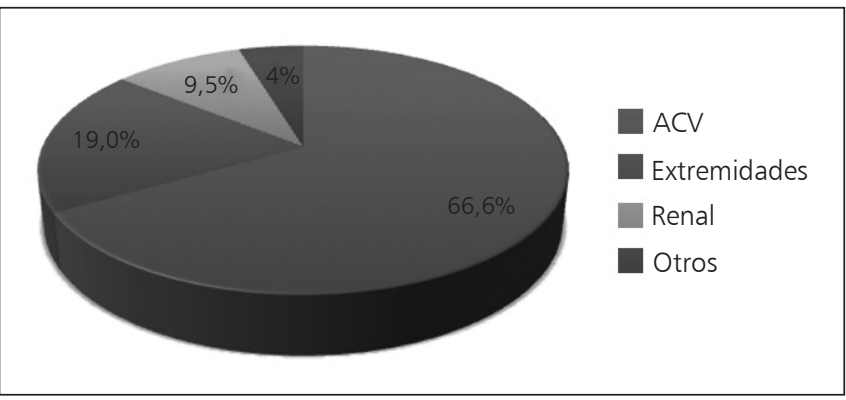

Figura 3. Eventos embólicos $(n=21)$.

Tabla 4. Condición de egreso

\begin{tabular}{|lccc|}
\hline Variable & Fallecidos & Vivos & p \\
\hline Sexo masculino & $19(65,5 \%)$ & $61(78,2 \%)$ & 0,13 \\
\hline Edad (años) & $55,2 \pm 2,3$ & $47,9 \pm 1,9$ & 0,04 \\
\hline FE ingreso I-II & $9(31,0 \%)$ & $38(49,3 \%)$ & 0,001 \\
\hline APACHE II* & $11,4 \pm 5,8$ & $6,6 \pm 3,6$ & 0,001 \\
\hline SOFA** & $4,7 \pm 3,8$ & $1,9 \pm 2,0$ & 0,001 \\
\hline Falla cardiovascular & $23(63,8 \%)$ & $13(36,1 \%)$ & $<0,001$ \\
\hline SDRA*** & $30(76,9 \%)$ & $9(23,0 \%)$ & $<0,001$ \\
\hline Días hospitalización & $17,3 \pm 13,1$ & $32,4 \pm 19,5$ & 0,002 \\
\hline Acidosis metabólica & $15(65,2 \%)$ & $8(34,7 \%)$ & $<0,001$ \\
\hline
\end{tabular}

*Acute Physiology And Chronic Health Evaluation. **Sepsis-related Organ Failure Assessment. ${ }^{* *}$ Síndrome distrés respiratorio agudo

\section{Complicaciones sistémicas}

La falla renal fue la más frecuente (Figura 2) y $8,4 \%$ requirió de diálisis aguda. En segundo lugar estuvo la insuficiencia cardíaca grave.

El 37,3\% de los pacientes requirió cirugía, efectuándose principalmente recambio valvular, cuyo motivo fue una insuficiencia aórtica o mitral agudas. La mediana de espera de cirugía fue de 9 días. La mortalidad quirúrgica fue de $27,5 \%$, destacando que la gran mayoría de estos pacientes se encontraban en CF III-IV previo a la intervención.

\section{Eventos embólicos}

El 19,6\% $(n=21)$ de los pacientes presentó fenómenos cardioembólicos, los que aparecieron a los 4,8 días desde el ingreso, siendo los accidentes cerebrovasculares los más frecuentes $(66,6 \%)$ (Figura 3).

\section{Evolución hospitalaria}

La media de tiempo de hospitalización fue de 28,3 $\pm 19,1$ días.

El 47,6\% de los pacientes debió ingresar a la Unidad de Cuidados Intensivos. El 21,5\% de los pacientes cursó con acidosis metabólica importante, $36,4 \%$ requirió conexión a ventilación mecánica, con un promedio de 5,9 días, 45,7\% requirió apoyo con drogas vasoactivas, con una mediana de uso de 4 días y 27,1\% desarrolló una sepsis severa.

El 18,6\% de los pacientes presentó algún tipo de infección asociada a la atención intrahospitalaria, siendo la más frecuente la infección del tracto urinario.

Veintinueve fallecieron durante su estadía hospitalaria, lo que ocurrió a los 17,3 $\pm 13,1$ días, siendo su causa principal un shock cardiogénico. La mortalidad global de la serie fue de $27,1 \%$. Los pacientes que tuvieron sólo tratamiento médico fallecieron en el 30,1\% y los sometidos a tratamiento quirúrgico en el 25,6\%.

La Tabla 4 muestra las características de los pacientes fallecidos comparados con los que sobrevivieron. Los fallecidos eran principalmente de sexo masculino, 
de mayor edad (55,2 $\pm 2,3$ años vs $47,9 \pm 1,9$ en los pacientes vivos). Los scores de gravedad como la capacidad funcional, el APACHE II y SOFA se asociaron a un peor pronóstico. Los pacientes que fallecieron presentaron igualmente mayor falla cardiovascular, acidosis metabólica y Síndrome de Distrés Respiratorio del adulto, alcanzando significancia estadística en comparación a los pacientes vivos. La letalidad por s.viridans fue de $30 \%$ y la por estafilococo de $36 \%$.

\section{Discusión}

Tras las publicaciones de Oyonarte et al en los años $2003^{6}$ y $2008^{9}$, ésta constituye una de las mayores series de pacientes con EI reportadas en Chile. Aún cuando su información está circunscrita fundamentalmente a pacientes de la Región de la Araucanía, puede representar la de otros centros de derivación del país. Cabe destacar que el $71,1 \%$ de los pacientes eran procedentes de Temuco, mientras que el $28,9 \%$ provenía de las restantes comunas de la IX Región. Ello permite estimar una incidencia aproximada de 1,8 casos por cada 100.00 habitantes por año (considerando una población regional de 850.000 habitantes), similar a lo reportado en el pasado en la ciudad de Santiago ${ }^{6}$.

La existencia de casi $30 \%$ de pacientes de origen rural puede influir en el retraso del diagnóstico, la microbiología aislada, la instauración de tratamiento y el pronóstico posterior. La capacidad funcional al ingreso fue II-III en el 74,5\% y el puntaje APACHE II de 8,4 $\pm 4,7$.

La edad fue de 49,9 $\pm 16,4$ años, correspondiendo el $74,7 \%$ a sexo masculino. En general, la edad y el sexo tienen un impacto en la incidencia de EI. Más de la mitad de todos los casos de EI en los Estados Unidos de Norteamérica y Europa se producen en pacientes mayores de 60 años, y la edad media ha aumentado durante los últimos 40 años ${ }^{11}$. Esta tendencia se debe probablemente a la disminución de la incidencia de la enfermedad reumática ${ }^{5}$, como un factor de riesgo para EI, y a la creciente proporción de adultos mayores que desarrollan enfermedad valvular degenerativa ${ }^{12}$.

El promedio de edad en Chile y en nuestra serie ha sido claramente menor, probablemente por la persistencia de un mayor número de pacientes con daño reumático, muchas veces sin diagnóstico de certeza.
Los hombres predominan en la mayoría de las series, con una proporción hombre-mujer que va desde 3:2 a 9:1; y una incidencia en los hombres entre 8,6 a 12,7 casos por 100.000 años-persona, destacando un aumento en el grupo de mujeres de 1,4 a 6,7 casos por 100.000 años-persona en las últimas tres décadas ${ }^{13}$.

El $58,7 \%$ presentó comorbilidad cardiovascular, principalmente hipertensión arterial, en el $41,1 \%$ de los casos. El 16,4\% presentaba DM, lo cual está descrito como un factor independiente de mortalidad intrahospitalaria en pacientes con $\mathrm{EI}^{14}$.

Respecto a la enfermedad reumática, ésta alcanzó al 10,2\% en nuestra serie. En el Estudio Cooperativo Nacional de Endocarditis Infecciosa (ECNEI) se reportó una incidencia de 25,3\% de origen valvular reumático ${ }^{6}$ en los primeros 321 pacientes publicados (1998-2002), a lo que posteriormente se sumaron 185 pacientes correspondientes al período 2003-2006; completando un total de 506 pacientes, con una prevalencia final de $21,6 \%{ }^{30}$. Esto reafirma la tendencia decreciente de esta patología en el tiempo ${ }^{5}$, así como la diferencia de los criterios diagnósticos para daño valvular reumática, que fueron bastante restringidos en nuestro caso .

\section{Válvula afectada}

El compromiso en válvula nativa fue de $90,7 \%$. La válvula preferentemente afectada fue la aórtica (61\%) seguida de la válvula mitral $(49,5 \%)$, coincidiendo con otras series al respecto ${ }^{15,16}$. El número de endocarditis protésicas es cada vez mayor; así lo demuestra un estudio realizado por Braun et $\mathrm{al}^{17}$, donde alcanza un $28 \%$, mientras que en nuestra casuística se presentó en el 9,3\% de los casos. EI sobre válvula protésica se desarrolla en 1 a $4 \%$ de los pacientes en el primer año tras la cirugía y posteriormente en aproximadamente $1 \%$ por $\mathrm{anno}^{18}$.

\section{Hemocultivos}

En el 68,3\% de los pacientes se logró aislar germen en los hemocultivos, lo cual es mayor a otras series nacionales ${ }^{19}$. Esto destaca la importancia de contar con un óptimo laboratorio con cultivos automatizados. Parte del número de cultivos negativos obtenidos pudiese explicarse por el inicio de antibióticos previo a la toma de muestras en el 45,1\% de los casos. Tornos, en el registro europeo, reporta obtención de hemocultivos positivos en el $71 \%$ de sus pacientes 
Endocarditis infecciosa en Temuco, Chile - B. Stockins et al

antes iniciar tratamiento antibiótico ${ }^{16}$.

Series norteamericanas describen negatividad en sus cultivos entre 3-7\% Esto varía ampliamente de acuerdo a la población estudiada y al país en cuestión, con números disímiles que van del 12 al $56 \%{ }^{20,21}$.

\section{Ecocardiograma}

Constituye un pilar en el diagnóstico de la EI y está incorporado en los elementos a medir en los criterios de Duke. con valores de especificidad mayores a $95 \%$ y de sensibilidad cercanos al 70\%. En nuestra serie el ecocardiograma transtorácico fue positivo para la presencia de vegetaciones en casi el 75\% de los pacientes y permitió identificar complicaciones intracavitarias en una cifra significativa de ellos. El examen transesofágico fue el complemento diagnóstico, permitiendo diagnosticar un mayor número de vegetaciones y de abscesos.

La longitud de las vegetaciones valvulares en la ecocardiografía transtorácica, ha sido reportada como un fuerte predictor de nuevos eventos embólicos y mortalidad ${ }^{22}$.

\section{Microorganismos involucrados}

La microbiología ha ido variando en la frecuencia de los microorganismos aislados en las últimas décadas ${ }^{23}$, apreciándose un aumento en la incidencia de cepas estafilocócicas, bacilos gram negativos y gram positivos en diferentes series, incluidas algunas nacionales, lo que agrava el pronóstico de la enfermedad.

The International Collaboration on Endocarditis-Prospective Cohort Study identificó la microbiología en 2.781 pacientes de 58 centros en 25 países $^{24}$. Los estafilococos fueron el agente etiológico en 744 de 1779 (42\%) y los estreptococos en $712(40 \%)$. En dicha cohorte la mayoría de los pacientes tuvo EI sobre válvula nativa (72\%) y un $23 \%$ fue asociado a atención de salud ${ }^{25}$.

En nuestra serie, al considerar sólo los cultivos positivos, estos corresponden a S. viridans $(45 \%)$, S. aureus $(27,2 \%)$ y $S$. coagulasa negativo $(8,2 \%)$. El cultivo de otros gérmenes fue excepcional, lo que puede atribuirse a la dificultad en sus cultivos más que a su ausencia como germen etiológico.

\section{Complicaciones y cirugía}

Una complicación frecuente de la EI es la acentuación del daño valvular que lleva a insuficiencia cardíaca, alteraciones de la conducción, embolías a distancia y a la muerte ${ }^{26}$. El 37,3\% de los pacientes tuvo indicación quirúrgica, cuyo motivo principal fue la insuficiencia aórtica o mitral aguda con falla cardiaca aguda. En el registro ECNEI las principales causas de cirugía fueron complicaciones intracardiacas, insuficiencia cardiaca e infección persistente $^{6}$. Actualmente, la tendencia general respecto a la indicación quirúrgica tiende a ser cada vez más precoz y en mayor número de casos, llegando a cifras de 50\% de los pacientes en algunas series internacionales ${ }^{27}$.

En esta serie las complicaciones más frecuentes fueron: renal 53,2\% (con necesidad de diálisis en $8,7 \%$ del total de pacientes), cardiaca $33,6 \%$, deterioro neurológico $14,0 \%$, hepática $10,2 \%$. EL 19,6\% presentó fenómenos embólicos, ocurridos en una media de 4,8 días desde el diagnóstico, siendo la mayoría de tipo cerebrovascular.

\section{Letalidad}

El 27,1\% de nuestros pacientes falleció durante la estadía hospitalaria, la mayoría secundario a shock cardiogénico. Oyonarte y cols, el año 2003 describieron la mortalidad hospitalaria en alrededor de $29 \%{ }^{6}$, similar a lo descrito a nivel internacional con números que oscilan entre el 9,6 y $26 \%{ }^{10,28,29}$. En general la mortalidad tanto en EI en válvula nativa como en válvula protésica, está determinada por embolías al sistema nervioso central y/o deterioro hemodinámico.

La mortalidad de la afección según el tipo de gérmenes que la producen llega a: 4-16\% para estreptococo viridans; $15-25 \%$ para el enterococo, $25-47 \%$ para el estafilococo aureus y sobre el $50 \%$ cuando el agente infeccioso causal es la pseudomona aeruginosa, enterobacteriacias $u$ hongos ${ }^{1}$.

Cabe destacar la importancia de la infección por estafilococo aureus, por presentar mayor riesgo de: muerte ( 20 vs $12 \%$ ), presencia de eventos embólicos (60 vs 31\%), eventos de sistema nervioso central (20 vs $13 \%$ ) comparado con aquellos con infección por microorganismos diferentes, según los datos del ICE-MD (The International Collaboration on Endocarditis-Merged Database ${ }^{25,30}$. En nuestra serie el estafilococo tuvo una letalidad de $36 \%$, por encima de un $30 \%$ para el caso del estreptococo viridans. La ausencia de cultivo de germen no significó un riesgo de muerte diferente $(33 \%)$. 


\section{Pronóstico}

La literatura describe a la neumonia nosocomial, la edad avanzada, a los inmunocomprometidos, los diabéticos, el score APACHE II, la inestabilildad hemodinámica, alteración del estado mental y falla renal como predictores significativos de mortalidad ${ }^{31}$.

En nuestra serie los criterios de gravedad como la capacidad funcional y los índices de severidad APACHE II y SOFA fueron predictores de un mal pronóstico.

\section{Conclusión}

Se trata de una serie con un diagnóstico bien caracterizado que retrata la dificultad de un diagnóstico más precoz, vital para mejorar el pronóstico y con pacientes que ingresan ya en condiciones muy graves.

Respecto a limitaciones de éste, podemos mencionar la falta de seguimiento a largo plazo, debido principalmente a dificultades en el acceso por ruralidad, lo cual además influye en muchos de los factores mencionados anteriormente.

\section{Referencias}

1. Mylonakis E, Calderwood SB. Infective endocarditis in adults. N Engl J Med 2001; 345 (18): 1318-30.

2 .Wilson W, Taubert KA, Gewitz M, Lockhart PB, Baddour LM, Levison M, et al. Prevention of infective endocarditis: guidelines from the American Heart Association: a guideline from the American Heart Association Rheumatic Fever, Endocarditis, and Kawasaki Disease Committee, Council on Cardiovascular Disease in the Young, and the Council on Clinical Cardiology, Council on Cardiovascular Surgery and Anesthesia, and the Quality of Care and Outcomes Research Interdisciplinary Working Group. Circulation 2007; 116 (15): 1736-54.

3. Baddour LM, Wilson WR, Bayer AS, Fowler VG, Jr., Bolger AF, Levison ME, et al. Infective endocarditis: diagnosis, antimicrobial therapy, and management of complications: a statement for healthcare professionals from the Committee on Rheumatic Fever, Endocarditis, and Kawasaki Disease, Council on Cardiovascular Disease in the Young, and the Councils on Clinical Cardiology, Stroke, and Cardiovascular Surgery and Anesthesia, American Heart Association: endorsed by the Infectious Diseases Society of America. Circulation 2005; 111 (23): e394-434.
4. Habib G, Hoen B, Tornos P, Thuny F, Prendergast B, Vilacosta I, et al. Guía de práctica clínica para prevención, diagnóstico y tratamiento de la endocarditis infecciosa (nueva versión 2009) Versión corregida 26/01/2010. Rev Esp Cardiol 2009; 62 (12): 1465.

5. Tleyjeh IM, Abdel-Latif A, Rahbi H, Scott CG, Bailey KR, Steckelberg JM, et al. A systematic review of populationbased studies of infective endocarditis. Chest 2007; 132 (3): 1025-35.

6. Oyonarte M, Montagna R, Braun S, Maiers E, Rojo P, Cumsille JF. [Infectious endocarditis: morbi-mortality in Chile. Results of the National Cooperative Study of Infective Endocarditis (1998-2002)]. Rev Med Chile 2003; 131 (3): 237-50.

7. Bahamondes JC, Merino SG, Silva VA, Salman AJ, Redel SI, Droguett GJ. [Surgery for bacterial endocarditis: retrospective study of 32 patients]. Rev Med Chile 2008; 136 (1): 31-7.

8. Durack DT, Lukes AS, Bright DK. New criteria for diagnosis of infective endocarditis: utilization of specific echocardiographic findings. Duke Endocarditis Service. Am J Med 1994; 96 (3): 200-9.

9. Oyonarte M, Montagna R, Braun S. Endocarditis Infecciosa: Experiencia en 506 pacientes del Estudio Cooperativo Nacional en Endocarditis Infecciosa (ECNEI) y sobrevida a 10 años (1998-2008). Rev Chil Cardiol 2008; 27 (4): (Abstract)-442.

10. Chu VH, Cabell CH, Benjamin DK Jr, Kuniholm EF, Fowler VG, Jr., Engemann J, et al. Early predictors of in-hospital death in infective endocarditis. Circulation 2004; 109 (14): 1745-9.

11. Hill EE, Herijgers P, Claus P, Vanderschueren S, Herregods $\mathrm{MC}$, Peetermans WE. Infective endocarditis: changing epidemiology and predictors of 6-month mortality: a prospective cohort study. Eur Heart J 2007; 28 (2): 196-203.

12. Durante-Mangoni E, Bradley S, Selton-Suty C, Tripodi MF, Barsic B, Bouza E, et al. Current features of infective endocarditis in elderly patients: results of the International Collaboration on Endocarditis Prospective Cohort Study. Arch Int Med 2008; 168 (19): 2095-103.

13. de Sa DD, Tleyjeh IM, Anavekar NS, Schultz JC, Thomas JM, Lahr BD, et al. Epidemiological trends of infective endocarditis: a population-based study in Olmsted County, Minnesota. Mayo Clin Proc 2010; 85 (5): 422-6.

14. Kourany WM, Miro JM, Moreno A, Corey GR, Pappas PA, Abrutyn E, et al. Influence of diabetes mellitus on the clinical manifestations and prognosis of infective endocarditis: a report from the International Collaboration on Endocarditis-Merged Database. Scand J Infect Dis 2006; 38 (8): 613-9. 
Endocarditis infecciosa en Temuco, Chile - B. Stockins et al

15. Zalaquett R, Garrido L, Casas F, Morán S, Irarrázaval $\mathrm{MJ}$, Becker $\mathrm{P}$, et al. [Valve repair surgery in infectious endocarditis]. Rev Med Chile 2004; 132 (3): 307-15.

16. Tornos P, Iung B, Permanyer-Miralda G, Baron G, Delahaye F, Gohlke-Barwolf C, et al. Infective endocarditis in Europe: lessons from the Euro heart survey. Heart 2005; 91 (5): 571-5.

17. Braun S, Escalona A, Chamorro G, Corbalan R, Perez C, Labarca J, et al. [Infective endocarditis: short and longterm results in 261 cases managed by a multidisciplinary approach]. Rev MedChile 2000; 128 (7): 708-20.

18. Grover FL, Cohen DJ, Oprian C, Henderson WG, Sethi G, Hammermeister KE. Determinants of the occurrence of and survival from prosthetic valve endocarditis. Experience of the Veterans Affairs Cooperative Study on Valvular Heart Disease. J Thorac Cardiovasc Surgery 1994; 108 (2): 207-14.

19. Seguel E, González R, Alarcón E, Stockins A, Neira S. L, Aguilera D, et al. Factores asociados a mortalidad en cirugía de la endocarditis infecciosa en el Hospital Regional de Concepción entre 1985 y 2005. Rev Chil Cardiol 2008; 27 (4): 470-9.

20. Lamas CC, Eykyn SJ. Blood culture negative endocarditis: analysis of 63 cases presenting over 25 years. Heart 2003; 89 (3): 258-62.

21. Koegelenberg CF, Doubell AF, Orth H, Reuter H. Infective endocarditis in the Western Cape Province of South Africa: a three-year prospective study. QJM 2003; 96 (3): 217-25.

22. Thuny F, Di Salvo G, Belliard O, Avierinos JF, Pergola V, Rosenberg V, et al. Risk of embolism and death in infective endocarditis: prognostic value of echocardiography: a prospective multicenter study. Circulation 2005; 112 (1): 69-75.

23. Cabell CH, Jollis JG, Peterson GE, Corey GR, Anderson DJ, Sexton DJ, et al. Changing patient characteristics and the effect on mortality in endocarditis. Arch Int Med
2002; 162 (1): 90-4.

24. Murdoch DR, Corey GR, Hoen B, Miro JM, Fowler VG, Jr., Bayer AS, et al. Clinical presentation, etiology, and outcome of infective endocarditis in the 21st century: the International Collaboration on EndocarditisProspective Cohort Study. Arch Int Med 2009; 169 (5): 463-73.

25. Fowler VG Jr, Miro JM, Hoen B, Cabell CH, Abrutyn E, Rubinstein E, et al. Staphylococcus aureus endocarditis: a consequence of medical progress. JAMA 2005; 293 (24): 3012-21.

26. Wang A, Athan E, Pappas PA, Fowler VG Jr, Olaison L, Pare C, et al. Contemporary clinical profile and outcome of prosthetic valve endocarditis. JAMA 2007; 297 (12): 1354-61.

27. San Román JA, López J, Revilla A, Vilacosta I, Tornos P, Almirante B, et al. Rationale, design, and methods for the early surgery in infective endocarditis study (ENDOVAL 1): a multicenter, prospective, randomized trial comparing the state-of-the-art therapeutic strategy versus early surgery strategy in infective endocarditis. Am Heart J 2008; 156 (3): 431-6.

28. Hoen B, Alla F, Selton-Suty C, Beguinot I, Bouvet A, Briancon $\mathrm{S}$, et al. Changing profile of infective endocarditis: results of a 1-year survey in France. JAMA 2002; 288 (1): 75-81.

29. San Román JA, López J, Vilacosta I, Luaces M, Sarria C, Revilla A, et al. Prognostic stratification of patients with left-sided endocarditis determined at admission. Am J Med 2007; 120 (4): 369 e1-7.

30. Miro JM, Anguera I, Cabell CH, Chen AY, Stafford JA, Corey GR, et al. Staphylococcus aureus native valve infective endocarditis: report of 566 episodes from the International Collaboration on Endocarditis Merged Database. Clin Inf ect Dis 2005; 41 (4): 507-14.

31. McDonald JR. Acute infective endocarditis. Infect Dis Clin of North Am 2009; 23 (3): 643-64. 